\title{
Isolation of a non-haemadsorbing, non-cytopathic strain of African swine fever virus in Madagascar
}

\author{
M. GONZAGUE ${ }^{1}$, F. ROGER ${ }^{2}$, A. BASTOS ${ }^{34}$, C. BURGER ${ }^{1}$, \\ T. RANDRIAMPARANY ${ }^{5}$, S. SMONDACK ${ }^{1}$ AND C. CRUCIERE ${ }^{1 *}$ \\ ${ }^{1}$ AFSSA-Lerpaz, 22, rue Pierre Curie, BP67, 94703 Maisons-Alfort Cedex, France \\ ${ }^{2}$ CIRAD-EMVT, TA 30/G, Campus International de Baillarguet, 34398 Montpellier Cedex 5, France \\ ${ }^{3}$ ARC-Onderstepoort Veterinary Institute, Private Bag X5, Onderstepoort 0110, South Africa \\ ${ }^{4}$ Department of Zoology \& Entomology, University of Pretoria, Pretoria 0002, South Africa \\ ${ }^{5}$ Institut Pasteur de Madagascar, BP 1274, Antananarivo 101, Madagascar
}

(Accepted 15 November 2000)

\section{SUMMARY}

African swine fever (ASF) suspected clinically in Madagascar (1998-9) was confirmed by polymerase chain reaction (PCR) and nucleotide sequencing, following virus isolation. No haemadsorption or cytopathic effect could be detected following leukocyte inoculation, but viral growth in cells was confirmed by PCR. Detection of ASF virus genome was carried out by amplification of a highly conserved region coding for the p 72 protein. Nucleotide sequencing of the amplicon revealed $99 \cdot 2 \%$ nucleotide identity between the recent Malagasy strains and a virus recovered from the 1994 outbreak in Mozambique (SPEC265). A serological survey performed on 449 sera, revealed that only $5.3 \%$ of the sera taken from pigs between 1998 and 1999 were positive.

\section{INTRODUCTION}

African swine fever is an important disease of domestic pigs caused by an icosahedral double stranded DNA virus sharing characteristics with Iridoviruses and Poxviruses [1]. Recently [2], it has been classified within the Asfarviridae family, genus Asfivirus.

ASF was first recognized as a distinct disease in 1910 when domestic pigs were introduced in Kenya, where they could come in contact with wild swine. In 1957 the virus was found for the first time outside Africa, in Portugal, following which the virus was recorded in some European and American countries [3]. Today, the disease is eradicated in these countries, except among the wild boars in Sardinia. ASF is now confined in most countries of Sub-Saharan Africa.

ASF virus (ASFV), which is the only known DNA arbovirus, can infect hosts through either a sylvatic

* Author for correspondence. cycle or a domestic cycle [4]. In the sylvatic cycle, ASFV infects warthogs and bushpigs as well as ticks of the genus Ornithodoros (including Ornithodoros porcinus porcinus). Transmission from the sylvatic cycle to domestic pigs is most likely through feeding of infected ticks on pigs. Among domestic pigs, the virus may be transmitted through pork products in garbage fed to pigs but also by direct contact, carrier pigs, people and vehicles [5].

Clinical signs and lesions in case of ASF are very similar to those of classical swine fever so the laboratory diagnosis is very important for identifying ASFV. A traditional method of ASFV identification is the haemadsorption test [6]. In some cases, nonhaemadsorbing field strains have been observed, in which case other diagnosis tests such as the observation of cytopathic effect (CPE), pig inoculation and the ELISA test are used [7]. More recently, polymerase chain reaction (PCR) assay has been developed [8]. The ELISA test is the most widely used 
for detection of antibodies to ASFV and replaces indirect immunofluorescence. We report here the first ASFV outbreak in Madagascar, the characterization of the virus and the first epidemiological results.

\section{MATERIALS AND METHODS}

\section{Viruses}

Nord 1971 and Pyrénées 1974 recovered from pigs in France, Ivory Coast 1996 [9], Madagascar 1999/1, Madagascar 1999/2 [10,11] and Nigeria 1999 were isolated from pigs at our laboratory.

DRC (Zaire) 1977, Namibia 1989, SPEC 265 (Mozambique 1994), Benin 1997 were recovered from domestic pigs and were isolated at the Onderstepoort Veterinary Institute. As was RSA/W/1/99, recovered from a warthog (Phacochoerus aethiopicus) sampled in 1999 in South Africa. Kenya (Hinde II) 1959, Malawi 1978 and Tanzania (Kwh/12) were provided by the World Reference Laboratory, Pirbright, UK. Madrid 1970 was provided by the Instituto Nacional de Investigaciones Agrarias, Madrid, Spain.

ASFV Uganda (UGA) 1965, Spain 1970 (E70), Spain 1971 (BA71V) and Dominican R. (DR2) 1979, have been described before [12].

\section{Preparation of porcine leukocytes}

The culture of leukocytes was prepared by modification of the method described by Malmquist and Hay (1960). Briefly, $10 \mathrm{ml}$ of blood were discharged into a tube containing heparin and incubated for between 90 and $120 \mathrm{~min}$ at $37^{\circ} \mathrm{C}$ in a tilted position. The supernatant with the leukocytes was removed and centrifuged for $20 \mathrm{~min}$ at $2000 \mathrm{~g}$. The pellet was resuspended in Eagle medium to a final volume of $20 \mathrm{ml}$, supplemented with $10 \%$ pig serum obtained from the same animal. Approximately $2.5 \times 10^{6}$ cells per millilitre are obtained in this manner, which allows for the preparation of 15 tubes containing $2 \mathrm{ml}$ of cell suspension. Tubes were inoculated 3 days after preparation.

\section{Virus isolation}

Samples were thawed and ground with tissue culture medium containing antibiotics to make a 1:5 suspension. This suspension was centrifuged at $10000 \mathrm{~g}$ for $20 \mathrm{~min}$, and $0.5 \mathrm{ml}$ of the supernatant fluid was inoculated onto leukocyte cell cultures. Cultures were incubated at $37^{\circ} \mathrm{C}$ and examined for 6 days for haemadsorption and CPE. Negative samples were blind passaged five times.

\section{Double antibody sandwich immunoenzymatic assay for ASFV antigen detection}

For the antigen detection the Ingezim PPA Das kit (Ingenesa, Madrid, Spain) was used as described by the manufacturer.

\section{Blocking immunoenzymatic assay for antibodies detection to ASFV}

For the detection of antibodies, the Ingezim PPA Compac kit (Ingenesa, Madrid, Spain) was used as described by the manufacturer.

\section{Polymerase chain reaction (PCR) and sequence analysis}

The ASFV specific PCR procedure used to detect ASFV in samples and cell culture supernatants has been described in the working document of the Commission of the European Communities PVET/ EN/3160 [13]. The highly conserved region of the genome coding for the $\mathrm{p} 72$ protein was amplified with the following primers (given in $5^{\prime}-3^{\prime}$ orientation): Primer 1-ATGGATACCGAGGGAATAGC; Primer 2-CTTACCGATGAAAATGATAC.

The PCR products were sized by agarose gel electrophoresis against a molecular weight marker, prior to purification and nucleotide sequencing (Genome Express Society, Grenoble, France). Sequence analysis and sequence alignments were done with the J. Hein method [14] and phylogenetic trees were constructed using the Megalin program of Lasergene software (DNASTAR).

\section{RESULTS}

\section{Pathological and epidemiological observations}

In the early stages of the disease, the pigs consumed decreasing amounts of feed and then had a staggering gait followed by posterior paresis, wheezing, constipation and abortion for pregnant animals. Epistaxis was also often observed. The skin under the throat and on the ears developed areas of cyanosis and fever was repeatedly observed. The characteristic lesions were haemorrhagic diathesis. The overall mortality rate ranged between 80 and $90 \%$. Globally, the swine population in Madagascar decreased by $60 \%$. Acute 
Table 1. Detection of ASFV genomic DNA by PCR from tissue samples and of ASFV antibodies by ELISA from serum samples

\begin{tabular}{llll}
\hline \hline & Tissue samples & Serum samples & Serum samples \\
Province & $1998-9$ & $1996-7$ & $1998-9$ \\
\hline Antananarivo (Tananarive) & $22 / 24 \dagger$ & $0 / 90$ & $5 / 95$ \\
Toamasina (Tamatave) & $5 / 8$ & - & $11 / 121$ \\
Mahajanga (Majunga) & $2 / 2$ & - & $0 / 4$ \\
Antsiranana (Diego-Suarez) & - & - & $0 / 66$ \\
Fianarantsoa & $1 / 1$ & - & - \\
Toliara (Tulear) & $1 / 1$ & $0 / 5$ (Bushpig) & $0 / 12$ \\
\hline \hline
\end{tabular}

* Malagasy name (French name).

$\dagger$ Positive number/sample number.

and peracute forms were noticed. In most of the observed cases, adult pigs developed clinical signs before piglets.

\section{Detection of ASFV genomic DNA by PCR and sequencing}

Eighteen samples were initially received from Madagascar. PCR was performed on spleen and lymph node specimens, but not on faeces or nasal swabs. After PCR amplification, a single major band of approximately $280 \mathrm{bp}$ was observed, on agarose gel, for 3 of the 4 samples examined. The amplified products were characterized by nucleotide sequencing and compared to those obtained from identified viruses and published sequences (analysed in 'phylogenetic analysis').

A second batch of 56 samples (spleens and nodes), taken from 5 different areas was also received. PCR was performed on 36 samples, 31 of which were positive (Table 1, Fig. 1). These results were confirmed by the ELISA test.

\section{Virus isolation}

Neither haemadsorption nor cytopathic effect, could be observed by buffy coat cell inoculation, despite the proven susceptibility of the leukocyte cultures to infection with a control haemadsorbing virus. Virus growth 1 week after leukocyte inoculation was however demonstrated by PCR (Fig. 2).

\section{Phylogenetic analysis of ASFV Malagasy strain}

For phylogenetic analysis of ASFV, a portion of the highly conserved p72 gene was amplified and sequenced. By alignment of this sequence from Malagasy isolates with the same portion from published ASFV sequences, strains we had in our laboratory, strains isolated at Onderstepoort Veterinary Institute and strains provided by Pirbright Laboratory, we observed four groups (Fig. 3). In the second group, SPEC 265, a 1994 Mozambican isolate was most closely related to the Malagasy isolates. Zaire (DRC) 1977 and South Africa (RSA/W/1/99) 1999 were in the second subgroup. The aligned sequence identity was $99 \cdot 2 \%$ between the Malagasy isolates and the Mozambique 1994 virus (SPEC 265) and $98.3 \%$ between the Malagasy isolates and a Namibian isolate, falling within the second subgroup of the first group and which was near to Malagasy isolates. Between Malagasy isolates and African strains isolated in 1999, the identity was $97.9 \%$ (Fig. 4).

The comparison of 10 other Malagasy isolates from different areas and from different dates (Table 2) showed that all the Malagasy isolates were identical.

Among predicted amino acid sequence from this region of the genome, the identity was $100 \%$ between the isolates except for the UGA strain which had a leucine in place of a proline and the Malawi strain which had a threonine in place of an alanine and a glutamine in place of a proline.

\section{Detection of ASFV antibodies}

ASFV antibody detection was performed by ELISA. Initially, 27 sera from animals between 3 months and 5 years-old, were received. Three animals between 3 and 5 months old were positive. 


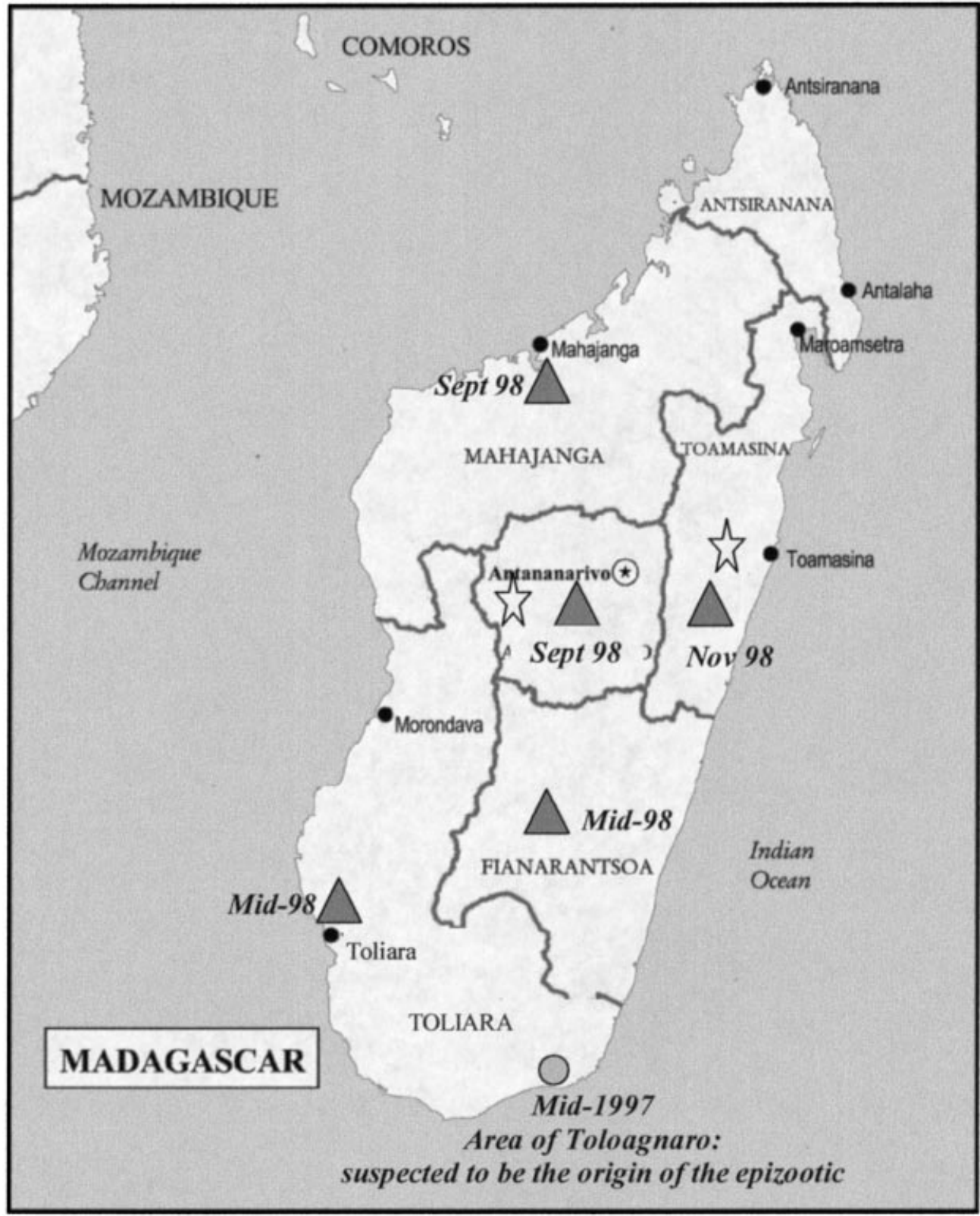

$\triangle$ : positive tissue samples for ASFV isolation

$\hat{W}$ : positive sera for ASFV antibodies

Date: date of the declaration of the first swine fever outbreaks

Fig. 1. Malagasy map with date of the first outbreak declaration in every province.

Subsequent to this, we received 90 sera taken from pigs in 1996. All were negative (Table 1). Among 298 sera taken from pigs between September 1998 and January 1999,16 sera $(5 \cdot 3 \%)$ were positive or doubtful. Five sera taken from bushpigs in 1996 were negative (Table 1).

\section{DISCUSSION}

The procedure routinely used to detect ASFV is the culture of suspected material on buffy coat cultures and observation of the haemadsorption induced by the virus. A few ASF virus isolates fail to haemadsorb [15] but still produce a cytopathic effect in the cells 


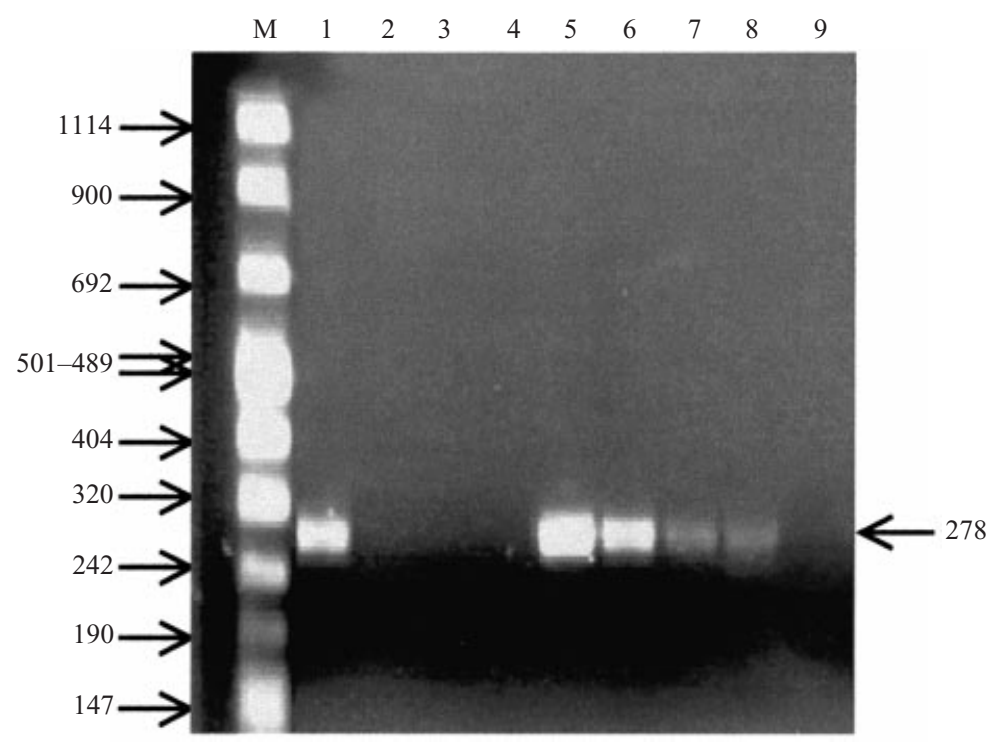

Fig. 2. In order to show that the non-haemadsorption and the non cytopathic effect were not in relation to no virus growth, PCR were performed on ASFV DNA extracted from equivalent volumes of virus containing cell culture medium and serial diluted. $\mathrm{M}$ : molecular weight markers, 1 to $4: 10^{-1}$ to $10^{-4}$ cell culture medium dilution directly after inoculum add. 5 to $9: 10^{-1}$ to $10^{-5}$ cell culture medium dilution 1 week post inoculation.

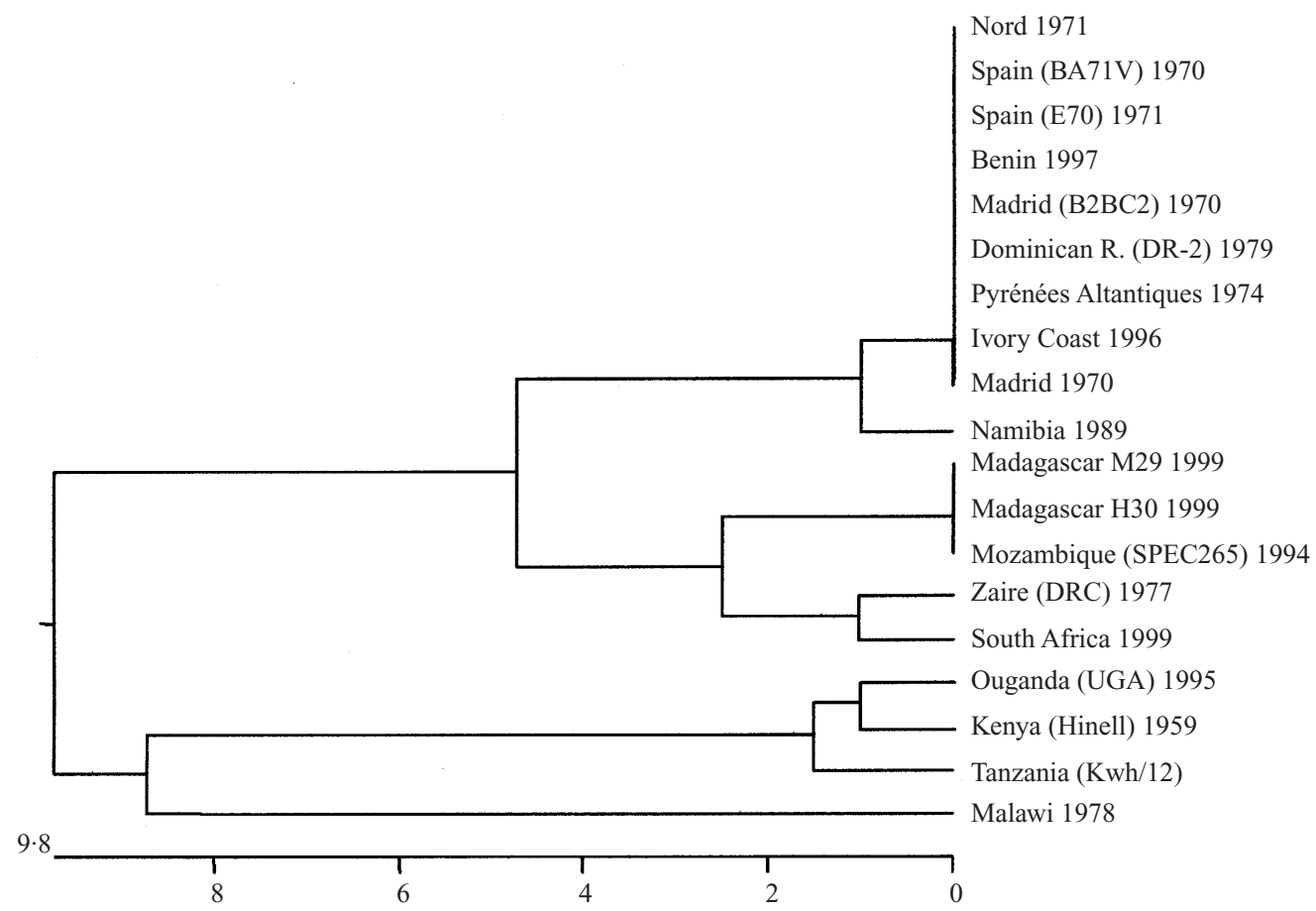

Fig. 3. Dendogram showing the genetic relatedness of Malagasy ASF virus isolates and published sequences. The phylogenetic tree was constructed from a part of the p72 gene.

used in the virus isolation [3]. The virus isolated from Malagasy pigs caused neither haemadsorption on initial cell passage nor cytopathic effect. Sanchez Vizcaino [8] observed that some samples from low virulent and chronic cases of the disease do not cause haemadsorption on initial cell passage. But, considering the syndromes produced by the ASF
Malagasy isolate, this is clearly not a low virulent strain, based on the definitions given in the manual of the European Communities. Wardley et al. [3] have suggested that antibodies may block the haemadsorption reaction but this seems unlikely in the Malagasy case in view of the number of animals with antibodies $(5 \cdot 3 \%)$ and the low antibody titres. 
GATACCGAGGGAATAGCAAGGTTCACGTTCTCGITAAACCAAAAGCGCAGCTTAATCCAGAGCGCAAGAGGGGGCTGATAGTATTTAGGGGITTGAGGTCCATTACAGCTGTAATGAACATTACGTCTTA Majority

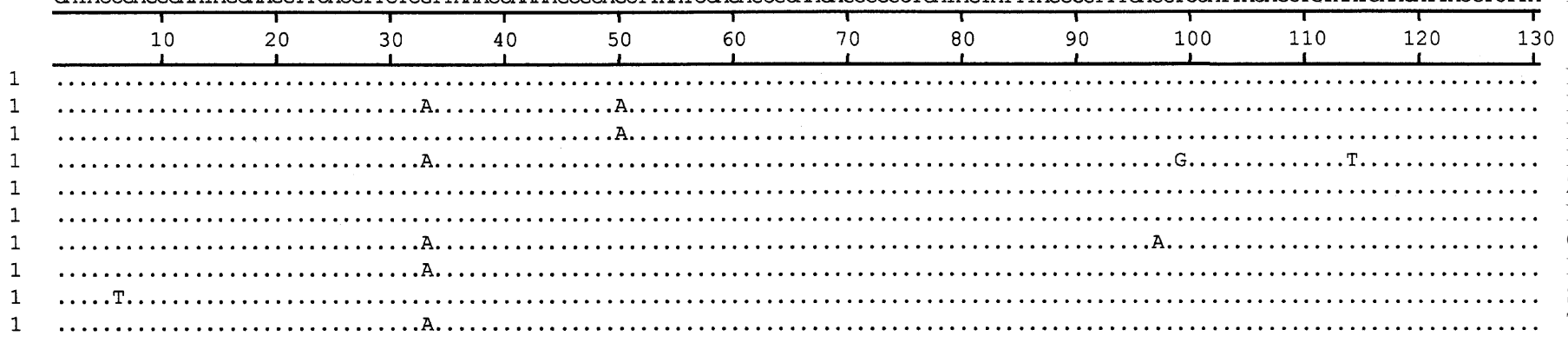

TGTCCAGATACGTTGCGTCCGTGATAGGAGTAATATCTTGTTTACCTGCTGTITGGATATTGTGAGAGTTCTCGGGAAAATGTTGTGAAAGAAATTTCGGGTTGGT

Nord 1971

Madagascar (M29) 1999

Mozambique (SPEC265) 1994

Malawi 1978

Zaire 1977

Namibia 1989

Ouganda (UGA) 1965

Kenya (HineII) 1959

South Africa 1999

Tanzania (Kwh/12)

$\begin{array}{llllllllll}140 & 150 & 160 & 170 & 180 & 190 & 200 & 210 & 220 & 230\end{array}$

Majority

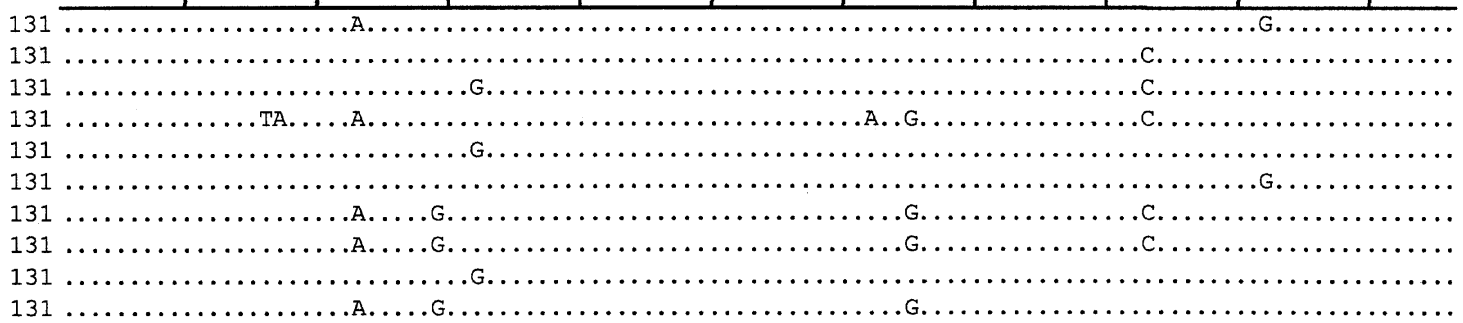

Nord 1971

Madagascar (M29) 1999

Mozambique (SPEC265) 1994

Malawi 1978

Zaire 1977

Namibia 1989

Ouganda (UGA) 1965

Kenya (HineII) 1959

South Africa 1999

Tanzania (Kwh/12)

Hide (as '.') residues that match the Consensus exactly.

Fig. 4. Sequence comparison of the p72 gene with one example of each phylogenetic group. Only the residues which differ from the concensus sequence are indicated. 
Table 2. Malagasy isolates from different areas and from different dates

\begin{tabular}{ll}
\hline \hline Areas & Dates \\
\hline Faretana (Antananarivo) & $11 / 01 / 1999$ \\
Mahajanga 1 (Maharanga) & $23 / 12 / 1998$ \\
Bevato (Maharanga) & $04 / 01 / 1999$ \\
Avaradrova (Tamatave) & $18 / 01 / 1999$ \\
Antanifotsy (Tamatave) & $18 / 01 / 1999$ \\
Faretana (Antananarivo) & no date \\
Tuléar (Toliary) & October 1999 \\
Andralanitra (Antananarivo) & $23 / 09 / 1998$ \\
Ambatolampy (Antananarivo) & March 1998 \\
Ambatofotsy (Antananarivo) & December 1998 \\
\hline \hline
\end{tabular}

Although the PCR was performed on a highly conserved part of the viral genome, sequence variation was observed between Malagasy strains, and viruses of European, American and African origin. The greatest identity was with SPEC265, a Mozambican virus recovered from the 1994 outbreak (99.2\%). With other temporally related African strains (South Africa (RSA/W/1/99) 1999 and Nigeria 1999), the identity was only $97 \cdot 9 \%$.

Bushpigs and the soft tick vector, Ornithodorus moubata, which are known to sustain infection in ASF endemic areas of Africa and to transmit the disease to domestic pigs $[16,17]$, are present in Madagascar $[11,18]$. The contact between bushpigs and domestic pigs is possible with free-ranging domestic pigs kept in the communal lands. However, the rapid spread of the virus among pigs, the peracute and acute forms of the disease and the serology (low titres and seronegative pigs prior to 1998) suggest that the disease may have been introduced recently either through swill from boat or plane, through pork products, or through live pigs. Epidemiological studies and sequence identity of the 12 Malagasy isolates, compared here, suggest a recent and single origin.

\section{ACKNOWLEDGEMENTS}

We wish to express our sincere thanks to $\mathrm{Dr}$ A. Ratovo (Direction des Services Vétérinaires of Antananarivo), Drs J. Domenech and A. Diallo (CIRAD-EMVT), Dr Hervé Zeller (Institut Pasteur of Madagascar. Drs T. de Ruyter and L. Bonneau (French Cooperation) for their assistance, and gratefully acknowledge the technical assistance of J. L. Edrich (University of Pretoria).

\section{REFERENCES}

1. Vinuela E. African swine fever virus. Curr Topics Microbiol Immunol 1985; 116: 151-70.

2. Pringle CR. Virus taxonomy. Arch Virol 1999; 144: 421-9.

3. Wardley RC, de M. Andrade C, Black DN, et al. African swine fever virus. Arch Virol 1983; 90: 73-90.

4. Kleiboeker SB, Burrage TG, Scoles GA, Fish D, Rock DL. African swine fever infection in the argasid host Ornithodoros porcinus porcinus. J Virol 1998; 72: 1711-24.

5. Mebus CA. African swine fever. Adv Virus Res 1988; 35: 251-69.

6. Malmquist AM, Hay D. Hemadsorption and cytopathic effect produced by African swine fever virus in swine bone marrow and buffy coat cultures. Am J Vet Res 1960; 21 : 104-8.

7. Sanchez-Vizcaino JM. Peste porcina africana. In: Servicio de Extension Agraria Ministerio de Agricultura, Pesca y Alimentacion, eds. Madrid, 1988.

8. Steiger Y, Ackermann M, Mettraux C, Kihm U. Rapid and biologically safe diagnosis of African swine fever virus infection by using polymerase chain reaction. J Clin Microbiol 1992; 30: 1-8.

9. Peste porcine africaine en Côte d'Ivoire. Informations sanitaires. O.I.E. 24 Mai 1996; 9 no. 19: 61.

10. Peste porcine africaine à Madagascar. Informations sanitaires. O.I.E. 15 Janvier 1999; 12 no. 1: 1.

11. Roger $\mathrm{F}$, Crucière $\mathrm{C}$, Randriamahefala $\mathrm{N}$, et al. African swine fever in Madagascar: epidemiological assessment of the recent epizootic. In Proceedings of the IXth International Symposium of Veterinary Epidemiology and Economics (ISVEE). Breckenridge, Colorado, USA, 6-9 August 2000.

12. Yu M, Morrissy CJ, Westbury HA. Strong sequence conservation of African swine fever virus p72 protein provides the molecular basis for its antigenic stability. Arch Virol 1996; 141 : 1795-802.

13. Diagnostic procedures for the confirmation and differential diagnosis of African swine fever. Commission of the European Communities. Manual for laboratory diagnosis of African swine fever. V1/ 1796/95-EN, 1995.

14. Hein J. Unified approach to alignment and phylogenies. Methods Enzymol 1990; 183: 626-45.

15. Pini A. Isolation and segregation of non-haemadsorbing strains of African swine fever virus. Vet Rec 1976; 99: 479-80.

16. Thomson GR. The epidemiology of African swine fever: the role of free-living hosts in Africa. Onderstepoort J Vet Res 1985; 52: 201-9.

17. Anderson EC, Hutchings GH, Mukarati N, Wilkinson PJ. African swine fever virus infection of the bushpig (Potamochoerus porcus) and its significance in the epidemiology of the disease. Vet Microbiol 1998; 62: $1-15$.

18. Uilenberg G, Hoogstraal H, Klein JM. Les tiques (Lxodoidea) de Madagascar et leur rôle de vecteur. Arch l'Inst Pasteur Madagascar. Numéro spécial, 1979. 